\title{
THRUST VECTORING EFFECTS OF A TRANSVERSE GAS INJECTION INTO A SUPERSONIC CROSS FLOW OF AN AXISYMMETRIC CONVERGENT-DIVERGENT NOZZLE
}

\author{
V. Zmijanovic ${ }^{1}$, V. Lago ${ }^{1}$, L. Leger ${ }^{1}$, E. Depussay ${ }^{1}$, \\ M. Sellam², and A. Chpoun ${ }^{2}$ \\ ${ }^{1}$ C.N.R.S. - Institute ICARE \\ $1 \mathrm{C}$ Av. de la Recherche Scientifique, Orléans cedex 45071, France \\ ${ }^{2}$ L.M.E.E., Université d'Evry Val d'Essonne \\ 40 rue du Pelvoux, Evry cedex 91020, France
}

\begin{abstract}
The transverse gas injection into the main supersonic flow of an axisymmetric convergent-divergent (C-D) propulsive nozzle is investigated for the fluidic thrust vectoring (FTV) possibilities as the segment part of the CNES "Perseus" project. Truncated ideal contour and conical C-D nozzles with different position and angle of the secondary circular injection port are selected as test models in the current numerical and experimental study. Analytical approach revealed parameters which affect the FTV efficiency, these criterions are further numerically explored and results data of the conical nozzle test cases are compared and coupled with the ones from experiments. It is found that upstream inclined injection has positive effect on vectoring capabilities and that with moderate secondary to primary mass-flow ratios, ranging around $5 \%$, pertinent vector side force is possible to be achieved.
\end{abstract}

\section{NOMENCLATURE AND ABBREVIATIONS}

2D two-dimensional

$3 \mathrm{D}$ three-dimensional

$C_{d}$ discharge coefficient 
CFD computational fluid dynamics

CFL Courant-Friedrichs-Levy

CPS code pour la propulsion spatiale (code for space propulsion)

$F_{a} \quad$ axial thrust force

$F_{j} \quad$ second injection reactive force (dynalpy flux)

$f_{m}=m_{j} / m_{i}$ mass-flow ratio

$F_{w} \quad$ force on the nozzle wall

$F_{x, y, z} \quad$ force components in $x, y$, and $z$ directions

FSS free shock separation

FTV fluidic thrust vectoring

$g_{0} \quad$ standard gravity acceleration

$h \quad$ injectant plume Mach disk height (distance from the nozzle wall)

$I_{\mathrm{sp}} \quad$ specific impulse

M Mach number

$m_{i} \quad$ primary mass-flow rate

$m_{j} \quad$ secondary mass-flow rate

$\mathrm{NPR}=p_{c} / p_{a}$ nozzle pressure ratio

$P_{0} \quad$ main flow total pressure

$p_{a} \quad$ ambient pressure

$p_{c} \quad$ chamber stagnation pressure

$p_{j} \quad$ second injection pressure

$p_{p} \quad$ plateau pressure

$p_{s} \quad$ stagnation pressure

$p_{\text {sep }} \quad-$ pressure at separation point

PDV primary downstream vortex

PUV primary upstream vortex

$q \quad$ dynamic pressure

SDV secondary downstream vortex

SITVC secondary injection thrust-vector control

$\mathrm{SPR}=p_{j} / p_{c} \quad$ secondary pressure ratio

SUV secondary upstream vortex

SVC shock vector control

TIC truncated ideal contour

TVC thrust vector control

$x$

$x_{j} \quad x$-coordinate of the secondary injection port

$x$-dimension along the nozzle axis from the throat

$x_{t} \quad x$-length of nozzle divergent section

$x / x_{i} \quad$ dimensionless $x$ coordinate

$y+=u y / \nu \quad$ dimensionless wall distance

$\gamma=C_{p} / C_{v} \quad$ heat capacity ratio

$\delta \quad$ pitch thrust-vector deflection angle

$\theta \quad$ injection angle with respect to nozzle axis

$\varphi \quad$ circular arc central angle 


\section{INTRODUCTION}

The sonic injection into the oncoming supersonic stream represents problematic at various aerospace engineering applications such as combustion in the scramjet engines and related issues with hypersonic vehicle reaction jets control. Properties and basic features of the supersonic cross flows are also the issue in FTV systems. The perspective of the thrust vector control (TVC) via fluidic means is of the special interest to the small space vehicles and systems. The elimination of robust actuators of the conventional mechanical TVC largely reduces mass of the system and simplifies design utilizing only the fast-opening valves and gas supply system. One order faster dynamic response of the FTV system than the conventional TVC $[1,2]$ and significantly smaller loss in the thrust specific impulse additionally emphasize FTV as an attractive alternative in thrust vectoring of a rocket engine. These characteristics are inline with the needs of a small launching vehicle, satellite's attitude control, or docking modules. The CNES "Perseus" project is aimed at the development of the smallscaled launcher systems among which optimal solution should be found for a cost-effective launch of small "micro" satellites. The promising large reduction in mass and size, simplicity, fast response, and possible effectiveness are highly beneficial to the vector control system of the small launchers intended by the CNES "Perseus."

There is a number of different techniques and modes of the fluidic thrust vectoring as the Coanda effect counter and co-flow injection control or skewing of the sonic line by fluidic displacement of throat surface [3, 4]. Among these various techniques, secondary gas injection thrust-vector control (SITVC) or the shock vector control (SVC) is selected as a direct and straightforward type of the FTV. In the investigations of two-dimensional (2D) planar nozzles reported in $[2-5]$, the high effectiveness of SITVC is affirmed but with cost of very considerable thrust reduction. The aim of the current study is to investigate secondary injection thrust vectoring effects and to optimize its application on the rocket type launcher nozzle.

\section{METHODOLOGY AND ANALYTICAL APPROACH}

In the current study, small to moderate mass-flow-rate ratios of around $\sim 5 \%$ are considered in order to reduce consequential effect on the regular rocket engine operation cycle and to preserve system efficiency. Experimental nozzle and vector system are tested under conditions for second and upper launcher stages using the conical type of C-D axisymmetric nozzles. The results are coupled and confronted with numerical data and results of analytical and empirical simplified models. After the comparison of the results and validation of the numerical 
procedure, truncated ideal contour (TIC) nozzle is numerically simulated as well by varying geometrical characteristics of the secondary injection.

\subsection{Physical Properties of Supersonic Cross Flows}

Strong bow shock generated by a secondary transverse injection at divergent (supersonic) portion of a nozzle is mainly responsible for diverting the nozzle jet. Resulting flowfield is characterized with the complex flow structures featuring strong adverse pressure gradients accompanied with the three-dimensional (3D) vortex and shock regions, boundary layer separation, shock generation, and their interaction, wakes, flow reattachment, and mixing shear layers.

The secondary injectant in the flow is acting as an obstacle and source of main jet momentum change. The upstream separation distance is in general determined by the flow nature of the boundary layer (laminar or turbulent) and by the penetration height of the injectant, as reported by Spaid and Zukoski [6]. Supersonic main flow "foresees" the secondary injectant plume as an obstacle and generates the bow shock as a response. In the basic case of supersonic cross-flow interaction, the turbulent boundary layer of the main flow detaches upstream of the injection port due to an adverse pressure gradient, which is the consequence of the bow shock, and it is followed by the weak separation shock (Fig. 1). Further downstream, separation shock is interacting with the strong bow shock. This interaction and the shock structure contribute in development of main flow deflection steeper gradient while between the shock region and the wall recirculation, shock bubble is formed. The structure between the wall, shock region, and the injectant plume (see Fig. 1) involves the counterrotating vortex pair, commonly known as the primary upstream vortex (PUV) which develops along the wall boundary and smaller counterrotating secondary upstream vortex (SUV) near the injectant plume. The separation shock formed along the displaced boundary layer by these vortices and sonic surface in between them are initially deviate the incoming flow. After separation region, main flow then faces strong bow shock and rapidly deflects. Sonically injected gas is underexpanded; thus, it is expanding in the main flow through the Prandtl-Meyer fan and is recompressed with the Mach disk at the end of this process.

On the downstream side of the injection, low-pressure region behind the jet creates suction which turns the injectant plume towards the wall. The lowpressure region is in greater part responsible for the wake which is dominated by strong vortical motions of primary and secondary downstream vortices (PDV and SDV). The closing edge of the pressure bubble on the downstream wall side, driven by the trailing edge of SDV and recompression shock reattaches the flow to the wall (see Fig. 1).

Spaid and Zukoski [6] constructed analytical model for prediction of the penetration height as a key parameter and pressure distribution in separated region. 


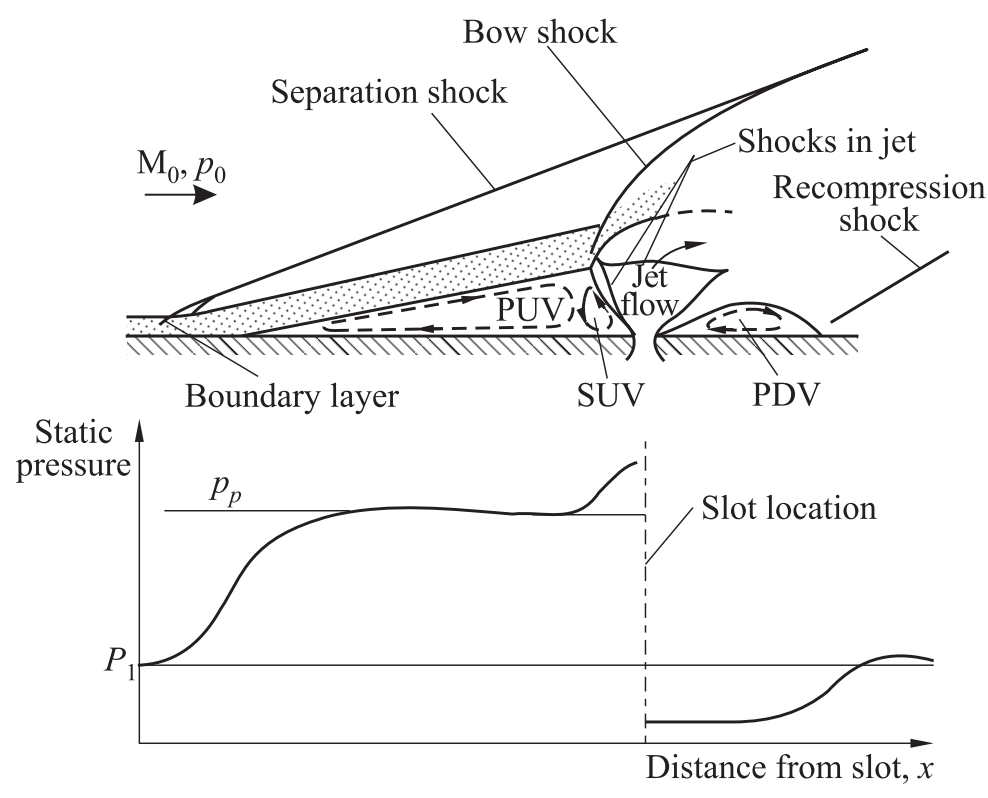

Figure 1 Two-dimensional flowfield and pressure distribution of transverse slot gas injection by Spaid and Zukoski [6]

Zukoski [7] at first observed that separated flow due to an injection acts in the quite similar manner as the flow that faces forward step on the wall. This model was further improved by Spaid and Zukoski blunt-body model with forward face of the step as a quarter of sphere with radius equal to the penetration height (Fig. 2).

Contrary to the slot injection at the flat plate, the circular injection exhibits $3 \mathrm{D}$ character. The physics of the $3 \mathrm{D}$ flow by this type of injection was before described via 2D analytical models and the empirical findings as in Schetz and Billig [8] and also in Avduevskii et al. [9].

With rapid development of computational fluid dynamics (CFD) methods and mathematical models, 2D and 3D supersonic cross-flows type are again broadly investigated and modeled since 1990s.

The model reported by Santiago and Dutton [10] and depicted in Fig. 3 also paved the way for numerical method of Chenault and Beran [11]. In this 3D case, formed flow structures demonstrate peculiarities in axial-vertical and spanwise lateral directions. The separated flow is initiated at the point or small region on a symmetry wall line and then it develops in 3D structure surrounding separated recirculation region. In the recirculation, two counter vortices PDV and SDV form 3D "horseshoe" vertical structure wrapping around the injection port. The 


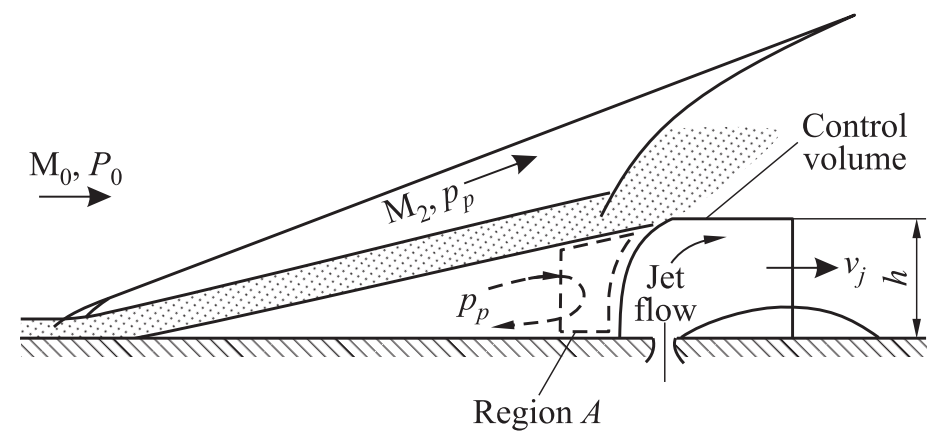

Figure 2 Blunt-body model of transverse injection into supersonic stream by Spaid and Zukoski [6]

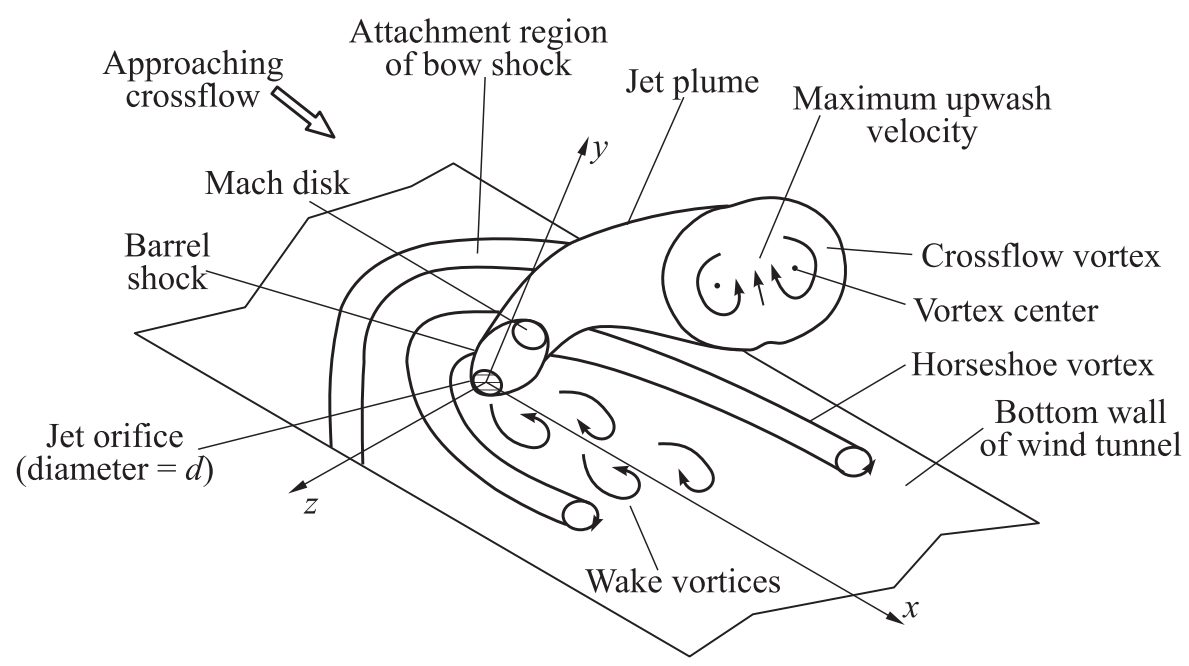

Figure 3 Circular gas injection at the flat plate into supersonic cross-flow by Santiago and Dutton [10]

strong bow shock propagates in the main flow in axial-vertical and spanwise direction bordering the main jet from the injectant plume which expands through the Prandtl-Meyer and is enclosed by the barrel shock. The physics of this $3 \mathrm{D}$ type of supersonic cross flow with more detail is described in [12].

Transverse sonic injection at the divergent part of the nozzle creates even more complex 3D flowfield involving bounded 3D nozzle flow and restricted shock propagation. The feedback loop forms between bounded nozzle flow and propagating flow structures. Bow shock lateral propagation is restricted with the 
nozzle wall. Therefore, on its outer ends, bow shock is pinched inward which reflects on the structures that bow shock encloses. Reversely, on the place where bow shock approaches the wall, boundary layer of the main flow detaches. This might inflict unsteadiness in the nozzle exhaust jet but also spanwise propagation allows pass for considerable amount of unaffected main flow and thus preserving adequate thrust efficiency.

\subsection{Analytical Models}

The analytical blunt-body model proposed by Spaid and Zukoski [6] has constituted a working basis for the development of the analytical model of a fluidic thrust vector performances calculation for supersonic C-D nozzle proposed by Sellam et al. [13].

As it was mentioned above, the gas injection into the divergent of a supersonic nozzle leads to shock interaction and separation zone upstream the injection hole that cause the deviation of the main nozzle flow by an angle $\delta$ (Fig. 4). Thrust vectoring effects induced by forces acting on the nozzle may be sorted as:

(1) pressure forces acting on the nozzle wall;

(2) viscous forces acting on the nozzle wall; and

(3) natural reactive force (momentum) of the secondary injection at the injection port.

The main jet deflection is inducing force imbalance in lateral direction generating the force on the wall in direction opposite to the deflection. The general lateral or vector force is further increased with the dynalpy flux or natural reactive force of the secondary jet.
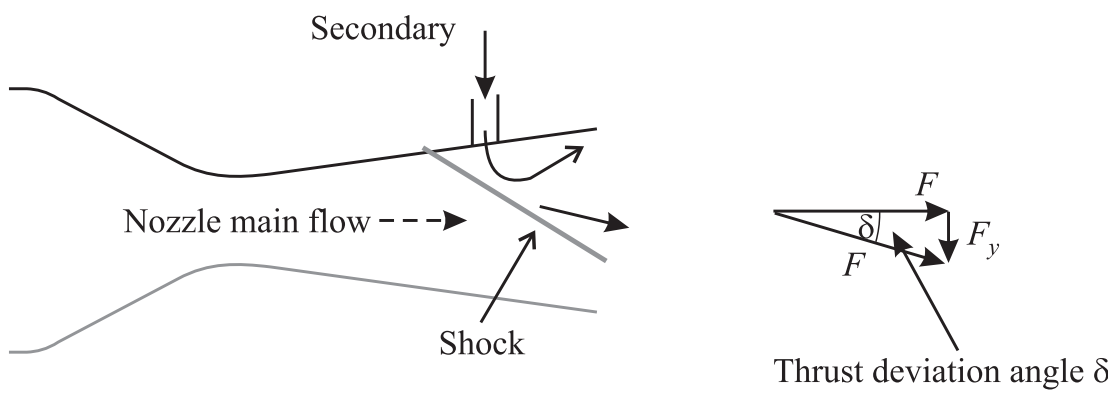

Figure 4 Scheme of the secondary injection thrust vectoring principle 
From geometrical considerations, one can easily write

$$
\delta=\tan ^{-1}\left[\frac{F_{y}}{F_{x}}\right]
$$

where $F_{x}$ and $F_{y}$ are the balance of forces along $x$ and $y$ direction, respectively.

The deviation angle $\delta$ is obtained from the pressure distribution downstream of the nozzle throat along the nozzle wall and from other parameters of primary and secondary flows (velocities and mass flow rates) as

$$
\delta=\tan ^{-1}\left[\frac{\sum f_{y}+\sum(\dot{m} U)_{y}}{\sum f_{x}+\sum(\dot{m} U)_{x}}\right]
$$

where $\Sigma f_{x}$ and $\Sigma f_{y}$ represent all pressure forces along the $x$ and $y$ axes and $\Sigma(\dot{m} U)_{X}$ and $\Sigma(\dot{m} U)_{y}$ represent all momentum fluxes acting along the $x$ and $y$ axes.

The analytical thrust vectoring model is mainly based on determining the shape of the line delimiting the separation area upstream the injection port (Fig. 5). The shape of this separation line is obtained from a correlation similar to that of Billig [14], initially built for the estimation of detached shock waves formed around spherical and cylindrical-nosed bodies. This correlation necessitates as input, the value of the flow separation distance $L_{s}$ along the stagnation line which can be evaluated by an iterative process once determined the height $h$ of the fluidic obstacle. In this approach, a constant plateau pressure $p_{p}$, deduced from a separation criterion, is applied along the line $L_{s}$, while a pressure gradient is estimated for the area between this line and the one bounded by the line of separation. This gradient varies, at each point $x_{k}$, from the plateau pressure $p_{p}$ to the pressure of the isentropic flow of an undisturbed nozzle at this abscissa $P\left(x_{k}\right)$ following a power-law formulation.

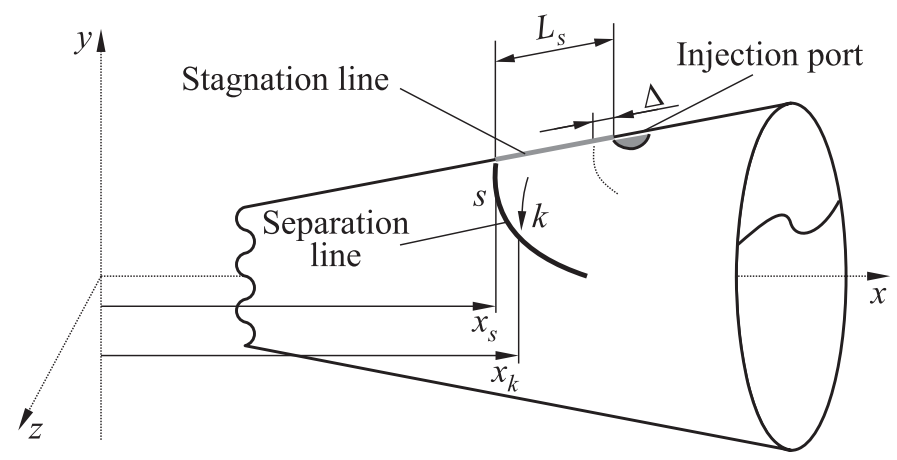

Figure 5 Wall traces topology of separation line 


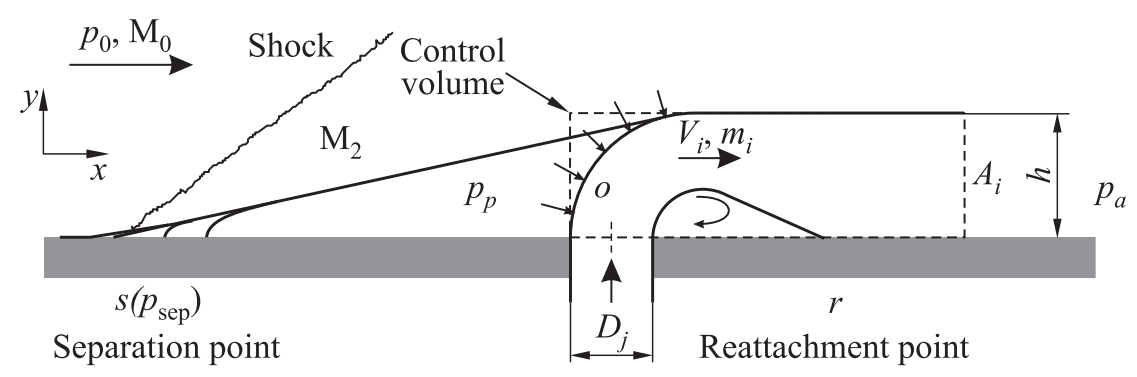

Figure 6 Simplified 2D scheme of secondary transverse injection and control volume [15]

The penetration height $h$ of the injectant can be estimated by applying a momentum balance in the $x$ direction to a control volume bounded by the interface between the main and the injected flows, the wall and the plane normal to the wall, downstream the flow reattachment point. This balance implies that the net pressure force, or drag, acting on the boundary surfaces of the control volume is equal to the momentum of fluid leaving the domain. For simplicity reasons, the interface between the main and the injected flows is assumed to be a quarter sphere of radius $h$ followed by a half open cylinder (Fig. 6).

The balance of forces is then given by the following equation in the $x$ direction:

$$
\sum F_{x}=\int \rho v^{2} d s .
$$

The sum on the left part of Eq. (1) can be calculated by decomposing the forces applied to the volume of control into two components:

$$
\sum F_{x}=F_{x 1}+F_{x 2}
$$

where $F_{x 1}$ is the pressure force applied on the interface of control volume; and $F_{x 2}$ is the pressure force applied on the back surface of the control volume.

The pressure force $F_{x 1}$, or drag, acting on the interface is given by integrating the pressure force over the spherical surface using the modified Newtonian law:

$$
F_{x 1}=\int_{0}^{\pi / 2} p d A .
$$

This integration gives:

$$
F_{x 1}=\frac{\pi h^{2}}{2}\left(p_{0}+\frac{1}{2} q_{0} C_{p \max }\right)
$$


where $C_{p \text { max }}$ is the pressure coefficient corresponding to the stagnation pressure $P_{01}$ behind the normal shock in the main flow at $p_{0}$ and $\mathrm{M}_{0}$ and $q_{0}$ is the dynamic pressure of the main flow at condition 0 before separation. $C_{p}$ max and $P_{01}$ are given by the following formulas:

$$
\begin{aligned}
C_{p \max } & =\frac{P_{01}-P_{0}}{\rho_{0} v_{0}^{2} / 2} ; \\
\frac{P_{01}}{p_{0}} & =\left[\frac{(\gamma+1)^{2} \mathrm{M}_{0}^{2}}{4 \gamma \mathrm{M}_{0}^{2}-2(\gamma-1)}\right]^{\gamma /(\gamma-1)}\left[\frac{1-\gamma+2 \gamma \mathrm{M}_{0}^{2}}{\gamma+1}\right] .
\end{aligned}
$$

The pressure force $F_{x 2}$ acts on the exit surface $A_{i}$ of the volume of control. If $p_{i}$ denotes the pressure that dominates this surface, then $F_{x 2}$ is given by

$$
F_{x 2}=-p_{i} A_{i}=-p_{i} \frac{\pi}{2} h^{2}
$$

The sum of forces is then given as

$$
\sum F_{x}=\pi h^{2}\left(\frac{p_{0}-p_{i}}{2}+\frac{1}{4} q_{0} C_{p \max }\right) .
$$

The secondary jet is sonically injected perpendicular to the flow, its momentum, after deflection in the $x$ direction, is the one obtained following an isentropic expansion:

$$
\int \rho v^{2} d s=\dot{m}_{j} V_{j}
$$

where $\dot{m}$ denotes the mass flow rate of the injected gas and $V_{j}$ the average flow velocity of the jet leaving the volume of control. The isentropic relationship gives

$$
\dot{m}_{j} V_{j}=2 C_{d} A_{j} \gamma_{j} p_{0 j}\left(\frac{2}{\gamma_{j}-1}\right)^{1 /\left(\gamma_{j}-1\right)}\left[\frac{1}{\gamma_{j}^{2}-1}\left(1-\left(\frac{p_{j}}{p_{0 j}}\right)^{\left(\gamma_{j}-1\right) / \gamma_{j}}\right)\right]^{1 / 2}
$$

where $P_{0 j}$ is the total pressure of the injected flow; $A_{j}$ is the injector section area; and $p_{j}=p_{i}$ is the stagnation pressure at the exit of the control volume.

By replacing the different terms in Eq. (1), one can easily evaluate the penetration height $h$ from the following relation:

$$
\begin{aligned}
h=D_{j}\left[C _ { d } \gamma _ { j } P _ { 0 j } ( \frac { 2 } { \gamma _ { j } + 1 } ) ^ { 1 / ( \gamma - 1 ) } \left[\frac{1}{\gamma_{j}^{2}-1}(1\right.\right. & \left.\left.\left.-\left(\frac{P_{j}}{P_{0 j}}\right)^{\left(\gamma_{j}-1\right) / \gamma_{j}}\right)\right]^{0.5}\right]^{0.5} \times \\
& \times\left[P_{0}-P_{i}+\frac{q_{0} C_{p \max }}{2}\right]^{-0.5}
\end{aligned}
$$


where $D_{j}$ an $C_{d}$ are the injection port diameter and the discharge coefficient, respectively.

Two empirical criteria adapted to conical nozzles are considered in analytical analysis. The Schilling criterion [16] based on experiments conducted with conical and truncated ideal nozzles gives the pressures relation for supersonic nozzle free shock separation (FSS) as

$$
\frac{p_{\text {sep }}}{p_{a}}=k_{1}\left(\frac{p_{0}}{p_{a}}\right)^{k_{2}}
$$

with $k_{1}=0.541$ and $k_{2}=-0.136$ as empirically found coefficients for conical type of nozzles and where $p_{0}$ and $p_{\text {sep }}$ denote, respectively, stagnation pressure of the oncoming flow near the separation and the wall pressure of the incipient separation point. It can be paired with the Green criterion [17] which gives the Mach number ratio through the oblique shock at separation. The $\mathrm{M}_{0}$ denotes Mach number of an oncoming supersonic flow before the separation and $\mathrm{M}_{2}$ behind the shock [15]:

$$
\frac{\mathrm{M}_{2}}{\mathrm{M}_{0}}=0.78 .
$$

After derivation for conical nozzles of 20 degree half-angle and for $\gamma=1.2$, Schilling provides equation to evaluate plateau pressure rise in the separation zone as a function of an increasing Mach number [16]:

$$
\frac{p_{0}}{p_{p}}=0.582\left(1+\frac{\gamma-1}{\gamma} \mathrm{M}_{0}^{2}\right)^{-0.1197 \gamma /(\gamma-1)} .
$$

\subsection{Geometry of the Secondary Injection}

In the presented experimental procedure, two conical nozzles with identical divergent sections and the two different second injection positions are investigated in the experiments; $x_{j} / x_{t}=0.7$ similar to the experimental case of Masuya et al. [18] and second one with injection at $x_{j} / x_{t}=0.9$. With the aim to test system without additional pumps and with low-moderate mass-flow ratio, secondary pressure ratio (SPR) is ranging up to unity value, while the secondary to primary mass-flow ratio is varying around 5\%. Additionally to these experiments, numerical simulations on the same test models are performed in order to couple and to compare the results and to obtain relations between the input values and the resulting vectoring capabilities. Using the method of characteristics, TIC nozzle of the same pressure ratio is designed and then corrected from numerical test results.

The conical convergent nozzle is used as gas injector geometry to transit the fluid from the small secondary chamber to the main nozzle. In the experiments 
and preliminary numerical simulations, transverse injection is normal to the nozzle axis. As it is reported by Nielson et al. [19] and Deere [4], upstream inclined injection has augmenting effects on the side force and vector deflection angle $\delta$. In order to investigate upstream inclined injection effect, injectors inclined upstream ranging from $20^{\circ}$ to $70^{\circ}$ are designed and numerically simulated.

\section{EXPERIMENTAL SETUP}

The experiments are performed at the CNRS institute ICARE "FAST" platform using the super/hypersonic wind-tunnel EDITH. The wind-tunnel is equipped, arranged, and setup by the authors. Wind-tunnel EDITH is set as the blowdown type wind-tunnel with test section of $1 \mathrm{~m}$ in diameter and $1.5 \mathrm{~m}$ in length: clean, oil-free, air is first dried, compressed till 300 bar and stored in 320-liter tank (Table 1). The air is supplied via 8-millimeter pipeline system to the pressure regulator and after regulation to the radial flow-splitter. Through the 6 radial distributed 8-millimeter tubes gas is supplied to the settling chamber of 160millimeter diameter and 200-millimeter length and exhausted through the nozzle to the test section of the tunnel. The model of the engine is with a lower chamber pressure of $300 \mathrm{kPa}$ exhausting to the depressurized till $8 \mathrm{kPa}$ test section and thus simulating the upper stages nozzle operation cycle. Two primary MPR ${ }^{\circledR}$ pumps with the power of $345 \mathrm{~kW}$ maintain the pressure in the test section of the wind tunnel which is adjusted through a butterfly valve (Fig. 7).

The primary experiments are conducted with two identical 5.42 degree halfangle conical type C-D nozzles with designed nozzle pressure ratio NPR $=37.5$, throat radius $R_{\mathrm{th}}=9.72 \mathrm{~mm}$, and expansion ratio $A_{\mathrm{th}} / A_{e}=0.236$. The circular $D_{j}=6 \mathrm{~mm}$ diameter injection port is normal to the nozzle axis. The secondary air is supplied through the 4 radial distributed tubes into the injectant settling chamber and from there smoothly via the convergent section to the sonic throat (see Fig. 7).

Table 1 The EDITH setup aerodynamic conditions

\begin{tabular}{ll}
\hline Stagnation conditions & Free stream conditions \\
\hline$p_{s}=300 \mathrm{kPa}$ & $p_{e}=8 \mathrm{kPa}$ \\
$T_{s}=245 \mathrm{~K}$ & $T_{e}=290 \mathrm{~K}$ \\
$\rho_{s}=2.3 \mathrm{~kg} / \mathrm{m}^{3}$ & $\rho_{e}=0.27 \mathrm{~kg} / \mathrm{m}^{3}$ \\
$\mathrm{NPR}_{D}=37.5$ & $U_{e}=615 \mathrm{~m} / \mathrm{s}$ \\
$A_{\mathrm{th}} / A_{e}=0.236$ & $\mathrm{M}_{e}=3.01$ \\
& $\mathrm{Re} / m=8,150,289 / m$ \\
& $\lambda_{e}=1.21 \cdot 10^{-7} \mathrm{~m}$ \\
\hline
\end{tabular}




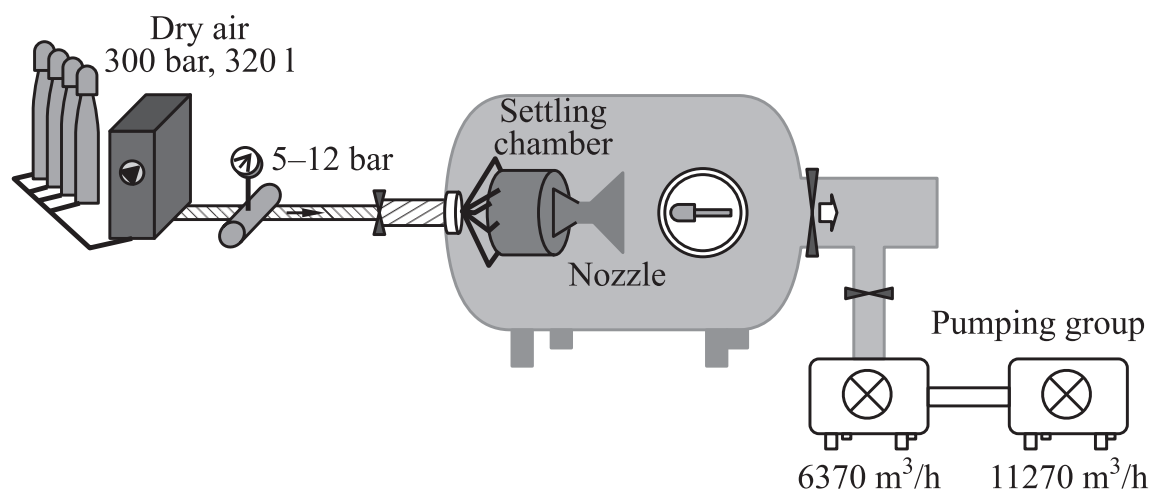

Figure 7 Scheme of the EDITH wind-tunnel operation cycle

The secondary dry air is supplied by the tubing system to the injectant settling chamber where the stagnation properties and the mass-flow rate are measured and recorded. From the secondary chamber air is smoothly via the converging nozzle injected into the nozzle.

The implemented diagnostic tools measure the flow properties: the stagnation pressure and temperature in the main and the secondary settling chambers, and also the ambient pressure and the temperature in the test section. Parietal wall pressure data is acquired via $11 \mathrm{Kulite}^{\mathrm{R}}$ XCQ-072 probes and via NI ${ }^{\circledR}$ DAQ SCXI fast acquisition cards. The probes are distributed along the wall iso-lines beginning from the line at injection port symmetry. Laterally they are distributed with intention to capture analytically predicted position of the separation (Fig. 8a).

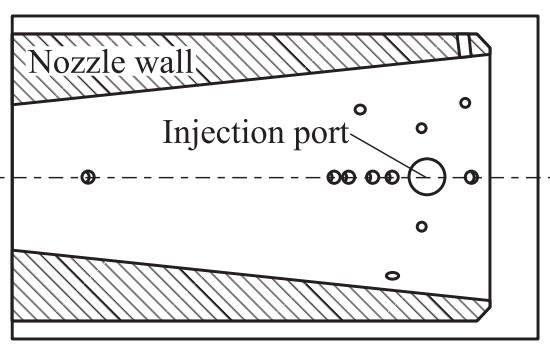

(a)

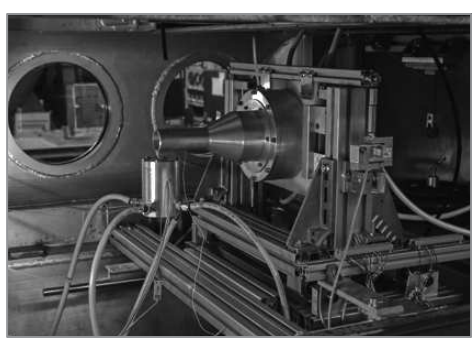

(b)

Figure 8 Conical nozzle CAD plan view $(a)$; and test system mounted on the balance with pressure probes distribution $(b)$ 
The $x, y$, and $z$ components of the force acting on the nozzle are measured using the force balance that is constructed by the authors. The test nozzle is placed on the very low or quasi-frictionless two-frame moving system which is connected to the four HBM force transducers bypassing signal via NI ${ }^{\circledR}$ SCXI DAQ acquisition and recoding force data. Two parallel placed transducers measure vertical or $Z$-force, one high-range transducers on the axial direction measuring axial or $X$-force and small-range transducer the lateral $Y$-force. The sensitivity of the small and higher range transducers is different as well the influence of the preload on the system. Therefore, the force balance is calibrated with fully mounted nozzle. Maximum and relative error margins and standard deviation are established on a number of test case loads. Additionally to these quantitative diagnostic tools, flow visualization Toepler's $Z$-schlieren is set up and also used. All the data are stored and processed with the Matlab $\left.{ }^{(}\right)$programs.

The experiments were performed under the adapted nozzle flow conditions and for three modes of overexpanded regime. The secondary injection pressure varied from 2 to 3.5 bar with 0.5-bar step and the test time was between 2 and $3 \mathrm{~min}$.

\section{NUMERICAL MODELS AND SIMULATION ENVIRONMENT}

In the current study, mass-averaged or Favre Navier-Stokes equations are solved with double-precision compressible solver of CPS_C. The CPS (Code pour la Propulsion Spatiale) is a $3 \mathrm{D}$ finite-volume CFD code designed and developed by Bertin, CNES, and SNPE for the space propulsive flows [20]. It is solving compressible multispecies reacting flows with fully accounted viscous effects on the unstructured 3D computational grid. Time splitting is used for explicit scheme of order up to 4 in time and up to 3 in space. The fluxes are computed on the cell interfaces with HLLC (by Toro) scheme for ideal gases and also for some preliminary cases using the Roe's upwind difference splitting scheme. In the present study, flux vectors are evaluated at each time step using the 2nd order scheme.

\subsection{Turbulence Models}

To model turbulent flows in the current study, the two-equation Launder-Jones $k-\varepsilon$ model is used. Provision is made for modeling boundary layer flows developing along the walls, in the presence of adverse pressure gradient. The wall function (adiabatic wall in the present study is imposed as a smooth wall) is coupled to the turbulence model by the procedure of modified logarithmic law of 
Van Driest [20]. Solver automatically disables wall function for $y+$ values below 10 which implies that for current study, wall function is mainly active near the walls in the external domain where the numerical grid resolution is lower.

Dry air is modeled as an ideal gas with power-law expressions for $\gamma(T)$ and $C_{p}(T)$ as a 7 th degree polynomials. Integration is achieved with fully explicit solver setting the time-step control ranging from unsteady for highest time accuracy to the steady optimized time step with Courant-Friedrichs-Levy (CFL) condition parameter in the solver up to 0.4 .

\subsection{Computational Domain and Boundary Conditions}

The 3D computational grid with one million of mapped hexagonal elements is built on the test-nozzle geometry consisting of 600,000 hex elements and exterior domain consisting of 400,000 hex elements (Fig. 9). The symmetry plane of the nozzle domain has computational grid $280 \times 100$. The grid density is clustered near the throat and towards the secondary injection port and slightly relaxed towards the exit. Exterior domain is clustered near the nozzle exit and then largely relaxed towards the outside boundaries. Size of the exterior domain is 15 nozzle exit diameters in the downstream direction, 2.5 diameters in the upstream direction, and 9 nozzle exit diameters in the radial direction. Boundary layer consists of 20 cells and additional 15 transitional cells with growth factor 1.14. The height of the first cell is $0.05 \%$ of the cross-section radius. In the present study, the $y+$ value is gradually devolving along the divergent section of the nozzle from 12 near the throat region to 4 at the exit section.

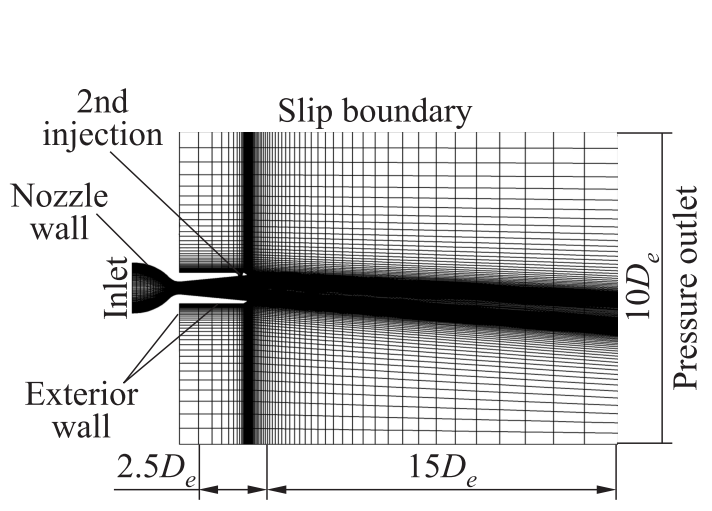

(a)

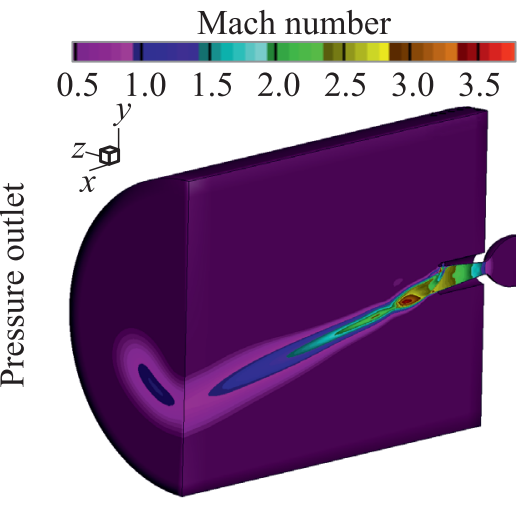

(b)

Figure 9 Computational grid (a); and 3D numerical domain with Mach number plot $(b)$. 
The injector port is modeled according to the test-model geometry as a convergent conical nozzle with 6 -millimeter throat diameter. This injector nozzle is perpendicular to the main nozzle $x$-axis as in the experimental test case and with the grid density clustered towards the injection port. Additionally to the basic two cases of normal circular injection, the cases with range of upstream inclined injector angles are numerically investigated as well.

To design the TIC nozzle, the code based on modified method of characteristics is built with potential velocity as the marching characteristic and the Sauer's method for calculation of the sonic line as the initial condition [21]. After the inviscid calculation and selection of the nozzle profile, several turbulence models that are available in CPS are used to test and correct preliminary nozzle profile for the boundary layer. The TIC nozzle is numerically investigated for two injector configurations: circular normal injection and with several angular upstream inclined injections.

\section{RESULTS}

\subsection{Flow Visualization and Qualitative Analysis}

In order to qualitatively analyze and determine the features in the flowfield produced by the secondary injection in the nozzle, schlieren results are considered and compared. Experimental $Z$-schlieren setup was able to acquire density gradient images in the external domain right after the nozzle exit. To complement these images with internal part of the flow, numerical schlieren is coupled together with experimental. Using the image processing in the Matlab ${ }^{\circledR}$, quasi-quantitative data of the deflection angle are obtained. Two identical experimental conical type C-D nozzles with injections at $x_{j} / x_{t}=0.7$ and 0.9 , respectively, demonstrated different flow characteristic and thrust vectoring effects for adapted nozzle operation and with SPR set to 1 .

Density gradients for the numerical schlieren depicted in Figs. $10 b$ and $11 b$ are calculated at the nozzle symmetry plane. The information of density gradients in experimental Toepler's $Z$-schlieren is acquired between the parabolic mirrors and projected via light beam. As a result of this all, the density gradient effects in lateral direction are superposed and projected on the plane. For the case with injection at $x_{j} / x_{t}=0.7$ in Fig. 10, it is observable that the bow shock propagates normally to the opposite side of the nozzle wall causing the shock wave reflection and separation of the boundary layer. The boundary layer through the recompression reattaches to the nozzle wall before the exit and forms the recirculation bubble between detachment and reattachment. This has severe effect on the vectoring and thrust performance as the reflection from opposite wall is opposing the flow deflection direction generated by the bow shock. Additionally, downstream of the injection port, there is enough wall length for the flow 


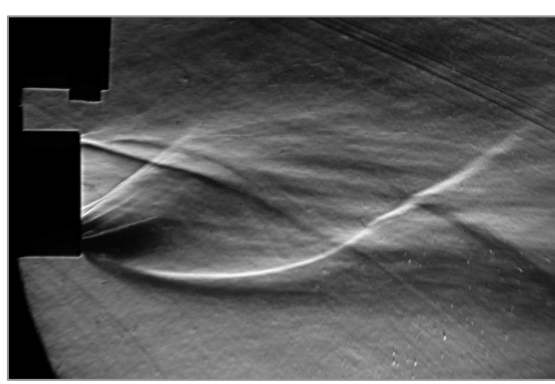

(a)

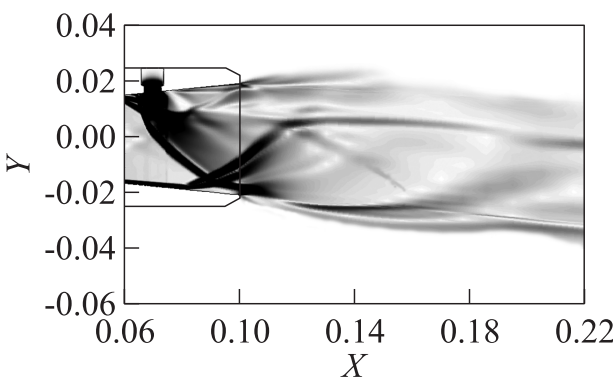

(b)

Figure 10 Experimental $Z$-schlieren $(a)$ and numerical schlieren $(b)$ at $x_{j} / x_{t}=0.7$ symmetry plane

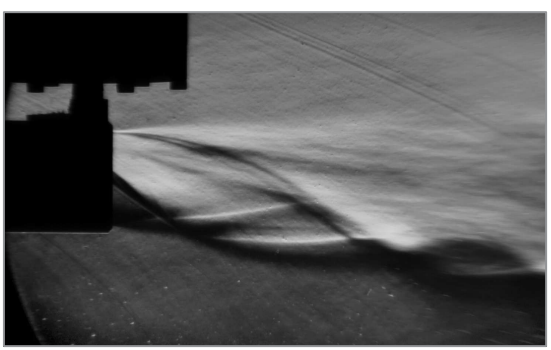

(a)

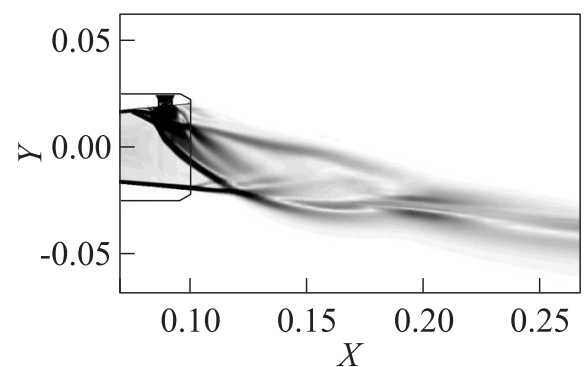

(b)

Figure 11 Experimental $Z$-schlieren $(a)$ and numerical schlieren $(b)$ at $x_{j} / x_{t}=0.9$ symmetry plane

to reattach which further negatively affects the nozzle performance and thrust vectoring.

The lines of the bow shock, reflected shocks, and the secondary jet plume exit the nozzle and are traceable on the experimental schlieren as well. The visualization measurement of the deflection pitch angle can be void since the flow in the nozzle plume after transverse injection is not homogenous. Therefore, these results are taken as the informative and as a reference in comparing the results from the force balance.

In the case of injection at $x_{j} / x_{t}=0.9$ (see Fig. 11), the bow shock, separated flow, and the jet plume exit the nozzle without wall interaction inside the nozzle. On the schlieren image in Fig. 11, it is also observable that the flow deflects with significantly larger angle.

In Fig. 12, iso-Mach surfaces depict 3D flow structures inside the nozzle generated by the transverse injection. The isosurfaces are accompanied with the 


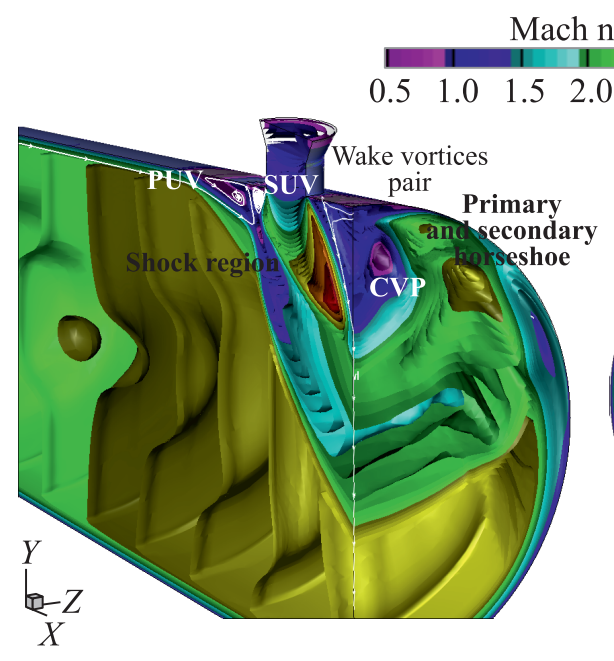

(a)

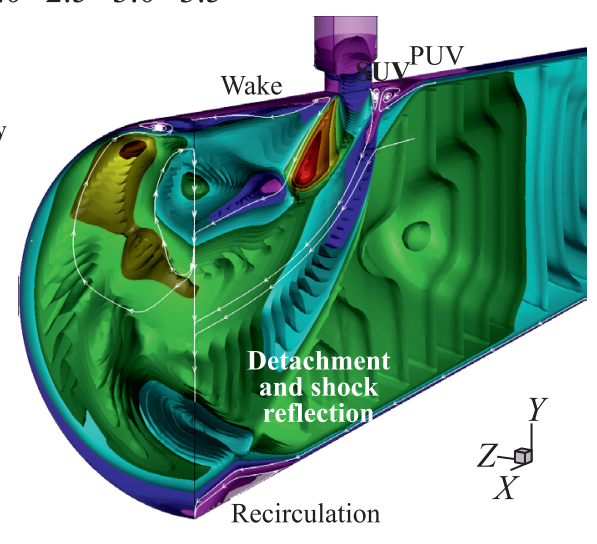

(b)

Figure 12 The 3D flowfields with iso-Mach contours of the $x_{j} / x_{t}=0.9(a)$ and $x_{j} / x_{t}=0.7$ secondary injection cases.

velocity streamtraces indicating the point of separation, PUV, SUV, and wake vortices. These vortices are forming $3 \mathrm{D}$ structures in shape of the horseshoe wrapping around the injectant plume as depicted in Fig. 12a. The wall effect of the nozzle on the bow shock pinches the outer envelope shock surface and the effect is than transferred on the shock region core and rest of the flow. Since the nozzle wall is axisymmetric, this deformation of the flow structures along lateral axis is symmetric.

The transverse injection placed deeper inside the nozzle (Fig. 12b) causes the reattachment on the opposite wall which is depicted on the schlieren images as well. As the bow shock which forms in the main flow surrounds the injectant plume, its propagation occupies completely nozzle cross section. Interaction of the bow shock with turbulent boundary layer on the opposite wall side separates the flow followed by the formation of recirculation shock bubble mechanism as described in [22]. Because of that, the complete main flow is forced to go through the bow shock which reduces the axial velocity of the nozzle jet and nozzle performance. Reattachments at the injection side surface and the closure of the recirculation bubble on the opposite wall side inflict the weaker shock reflections which are also traceable in Fig. $10 a$. The case of $x_{j} / x_{t}=0.7$ injection place has proper effects for highly or gross overexpanded nozzle regime as it is able to effectively act on the main flow and deflect it even with large separated zone in the nozzle due to an overexpansion. Also, it might be used for stabilizing the nozzle 


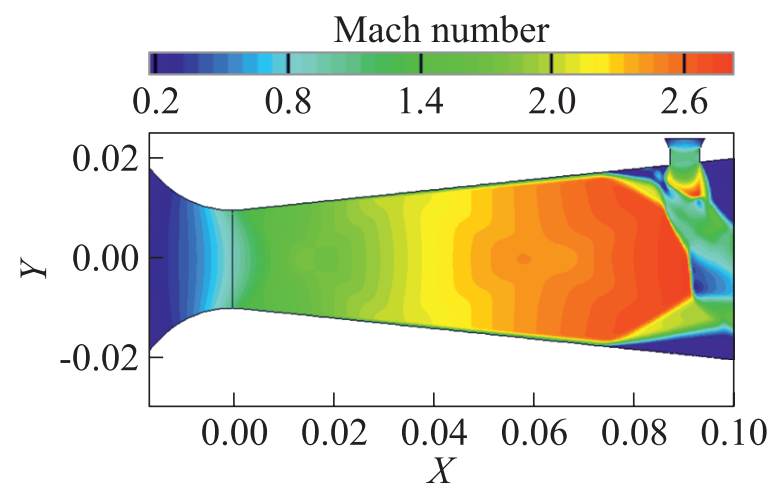

Figure 13 Mach number plot in the symmetry plane for overexpanded regime with $\mathrm{NPR}=7.5$.

flow in this regime by activating all injection ports. Otherwise, it is found that this regime due to additional effects negatively affects the nozzle with adapted or normal to high overexpanded operation regime. The high overexpansion case by lowering adapted NPR from 37.5 to 7.5 is depicted in Fig. 13. Several test cases with overexpanded regimes demonstrated that SITVC remains effective with considerably higher ambient pressures than the designed one.

The performance envelope starts to noticeably descend with regimes where flow separates inside the nozzle. Limiting case for thrust vectoring is found to be when the Mach disk appears at the injection port cross section of the nozzle. In the presented case in Fig. 13, it occurs for NPR $=7.5$ or 5 times less than the designed $\mathrm{NPR}_{D}=37.5$. Further increase of ambient pressure causes Mach disk to draw back inside the nozzle making the SITVC inoperable. Other than position of the injection port, the angle of injection and shape of the injection represent geometrical parameters that need to be considered in SITVC optimization process. As previously reported by Nielson et al. [19], it is found that secondary injection inclined upstream with respect to the nozzle axis has positive effect on the main flow deflection until some experimentally found breakdown value. Injection with upstream inclination angle causes "pushes" of the edge of a bow shock upstream inflicting stronger adverse pressure gradient and early separation (see Fig. 22 below). This generates rapid and steep deflection of the main jet which inflicts stronger force in lateral direction. The geometrical shape of injection reported by such authors as Deere [4], Wing and Giuliano [3], Guhse [23] unveiled that it has small positive, none, or negative effects on the thrust vectoring. While the "ring" shaped injection and multiple ports injection unveiled negative effects $[4,5]$, the slot injection had positive trend in thrust vectoring. 


\subsection{Flow and Wall Pressure Measurements}

In Fig. 14, wall-pressure profiles of the lower and upper wall edge of the nozzle symmetry plane are given. As previously denoted and reported by Chenault and Beran [11], it is possible to distinguish different regions in separated zone on the pressure profile. Region 1 is labeled as the region of steep pressure growth which is a consequence of boundary layer separation.

Region 2 marks pressure flattening and plateau pressure and it is caused by the PUV which is then followed by a pressure peak (region 3) imposed by the SUV and accompanied with secondary smaller "spike" caused by the injection sudden overexpansion. On the downstream side in region 4 , it is slight pressure rise is due to a leading edge of PDV and then pressure drop caused by the SDV. Pressure "hump" is closing this zone in region 5 , which ends with the pressure drop caused by a trailing edge of SDV, boundary layer reattachment, and recompression shock which can be detected in the case of injection at $x_{j} / x_{t}=0.7$. On the lower wall edge pressure profile, there is rapid pressure rise due to boundary layer detachment inflicted by the propagating shock. The pressure profile is flattened with recirculation formed inside the formed bubble and it ends with pressure drop caused by the recirculation trailing edge and recompression shock. These effects, as mentioned, have negative outcome on SITVC in terms of force, unsteadiness, and nozzle performance.

In the test case with injection at $x_{j} / x_{t}=0.9$ which is selected as the referent case, there is no reattachment on the upper edge since only PDV remains inside

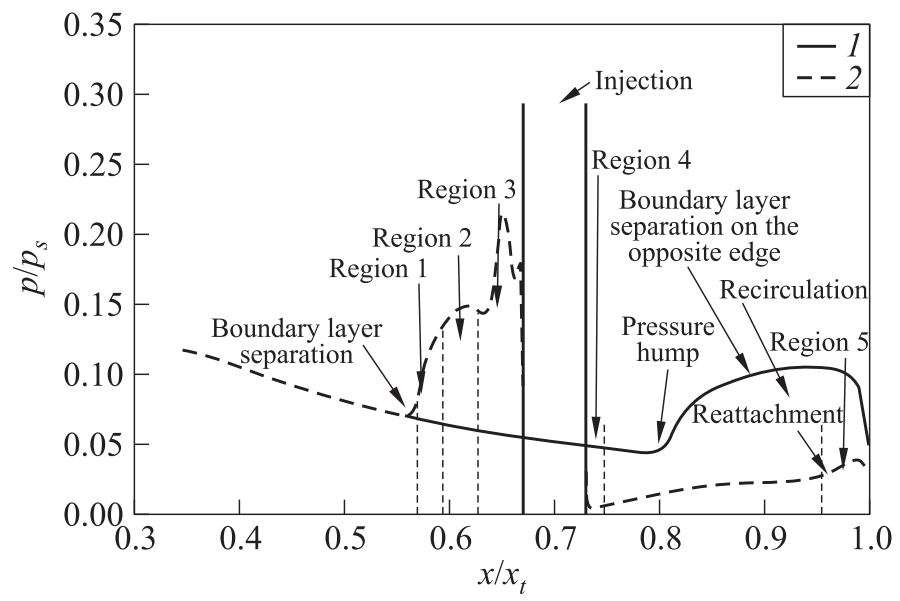

Figure 14 The $x_{j} / x_{t}=0.7$ injection case wall-pressure profiles in symmetry plane and distingue region notations at the lower (opposite) edge (1) and at the upper (injection) edge (2) of the nozzle symmetry plane 


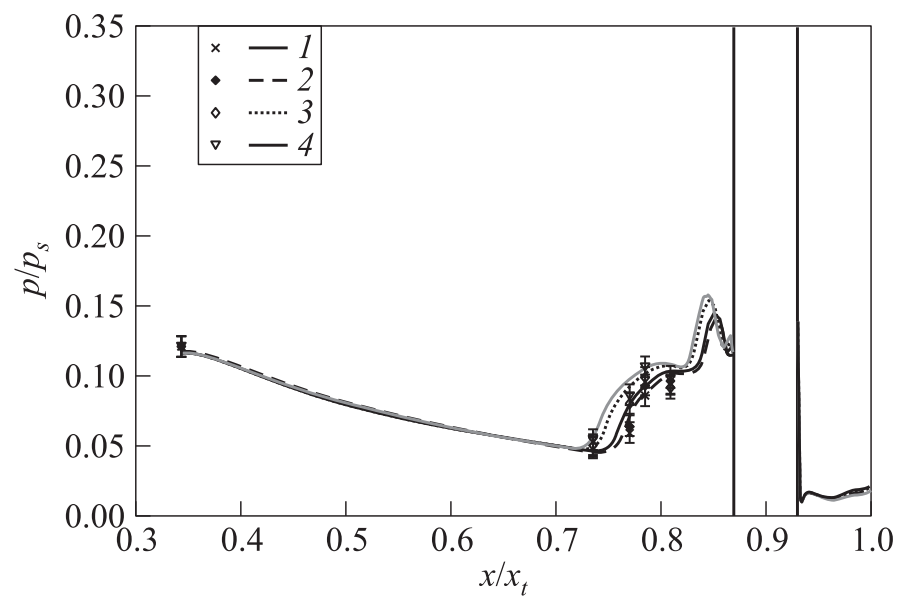

Figure 15 The $x_{j} / x_{t}=0.9$ wall-pressure for fixed NPR $=37.5$ and different secondary mass-flow-rate ratios $f_{m}: 1-0.055 ; 2-0.068 ; 3-0.080$; and $4-0.098$. Curves refer to numerical simulations and signs to experiments

the nozzle while SDV trailing edge is in exterior domain. The lower edge of the symmetry plane is unaffected from the transverse injection since the bow shock and the injectant leave the nozzle before impacting the opposite side of the wall.

The Kulite ${ }^{\circledR}$ pressure transducers which are placed in this test case took measurements in 2-second intervals with acquisition sample rate of 6000 samples per second. After the treatment of the signal and standard-deviation calculation procedure error margin of $2.8 \mathrm{mV}$ is found, this corresponds to $\sim 2 \mathrm{kPa}$ of pressure. The averaged pressure probe data with standard deviation is plotted together with the numerical data in Fig. 15. For the fixed adapted NPR $=37.5$ and varied SPR, presented in term of mass-flow-rate ratio, as depicted in Fig. 15, the mass flow rate ratio has great impact on the penetration height and thus, on the separation distance. The increase of secondary injection almost linearly affects separation with some difference between small and medium injectant mass-flow rates.

By fixing SPR = 1 and varying NPR from adapted to large overexpansion (Fig. 16), it is found that overexpansion has little or no influence on the upstream pressure profile. This is the case until the Mach disk passes nozzle cross section at injection port as depicted for NPR $=7.5$. In this case, separation moves a little backwards and some irregularities appear on the pressure profile in region 1. On the downstream part of the profile, there is a large pressure rise which depends on the ambient pressure. Calculating the penetration height defined with analytical procedure defined in subsection 2.2 , it is then possible to predict separation point pressure and plateau pressure by using the empir- 


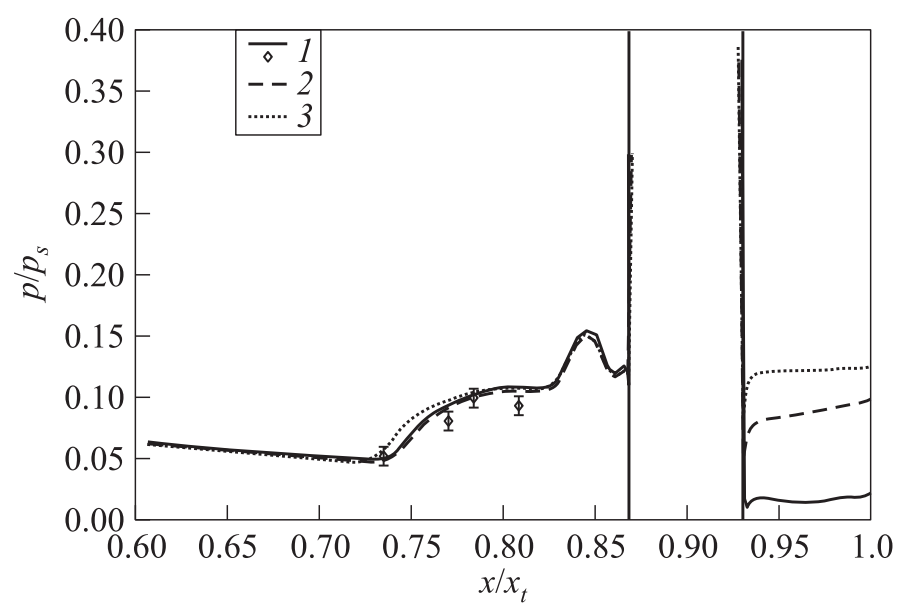

Figure 16 The $x_{j} / x_{t}=0.9$ wall-pressure for fixed SPR $=1, f_{m}=0.08$, and different NPR: $1-37.5$ (curve - numerical simulations and signs - experiments); $2-10$; and $3-7.5$

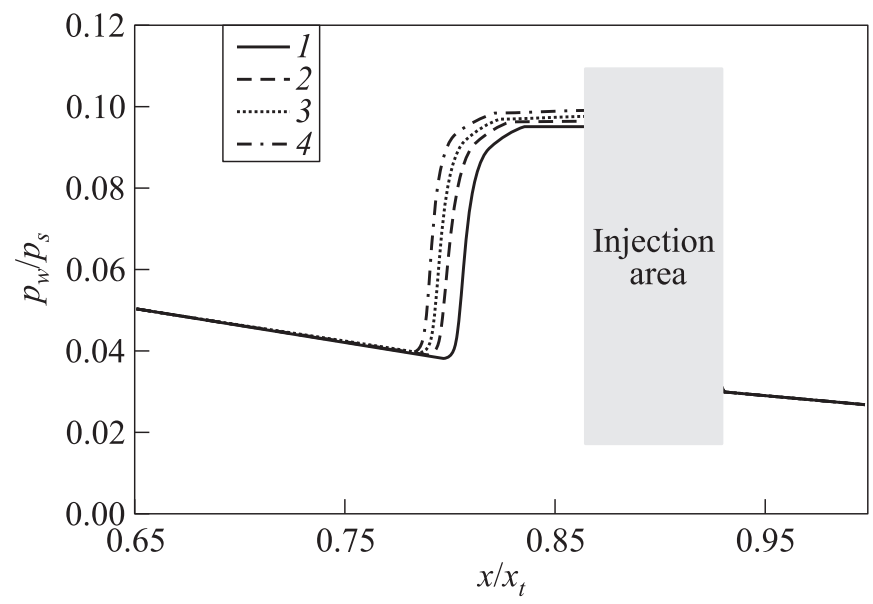

Figure 17 Profile build using the empirical criterion by Schilling for separation point and plateau pressure: $1-0.667 ; 2-0.883 ; 3-1.0$; and $4-1.167$

ical criterions. In Fig. 17, Shilling criterion is used together with Schmucker curve $[16,23]$ fitting the connect points of determined plateau and separation pressures.

Pressures determined by empirical and analytical formulations predicted and corresponded very well to the ones from CFD simulations and from the experi- 


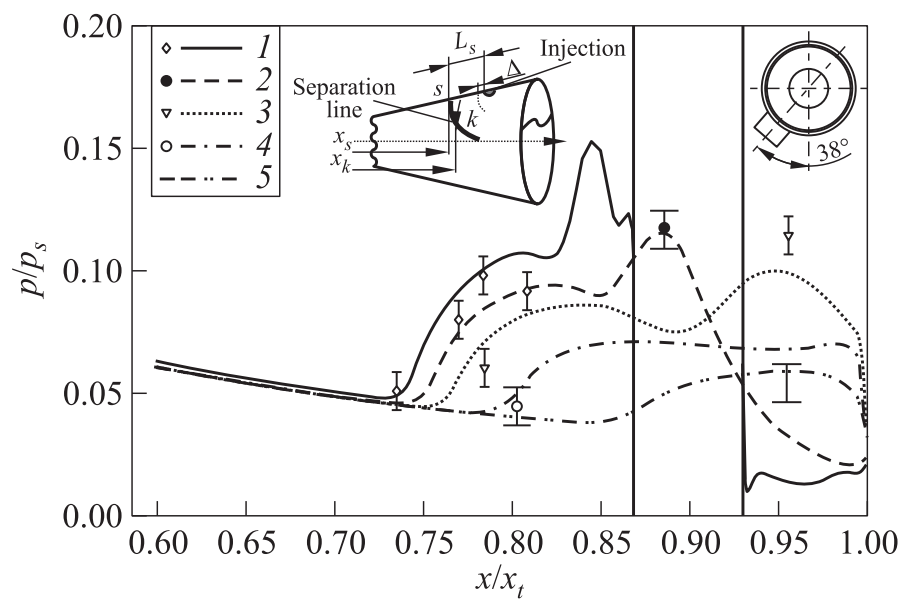

Figure 18 Wall-pressure profiles at radially distributed nozzle cross sections $(\mathrm{SPR}=1)$ and detection of the separation line: $1-0^{\circ}$ (symmetry); $2-25^{\circ} ; 3-38^{\circ}$; $4-60^{\circ}$; and $5-90^{\circ}$. Curves refer to numerical simulations and signs to experiments

mental results (Fig. 18). These analytically found pressure points were not able to predict movement in region 2 dominated by SUV and turbulent boundary layer effects but it was possible to use them in junction with analytical formulation of Schilling [16] and Green [17] for predicting approximate value of the vector pitch angle (Fig. 19).

Using the pressure data of the transducers and the Kulite ${ }^{\circledR}$ probes map in Fig. 6, it is possible to plot these results together with the numerical wallpressure data for a whole range of the nozzle horizontal cross sections around the nozzle axis: from $0^{\circ}$ to $90^{\circ}$. The results of this pressure profiles are analyzed together in order to detect the 3D separation line of the nozzle wall section.

It is possible to observe that regions dominated by PUV and SUV which form horseshoe 3D vortices wrap around the transverse jet, exit the nozzle and gradually dissipate along the late lateral nozzle-wall side. Applying the control on this flow mechanism by injection rate, angle and geometric shape of the injector is possible to affect the desired thrust vectoring performances.

\subsection{Force Measurement Data and Performance Analysis}

The measured vector side force is composed from pressure forces acting on the nozzle wall in lateral $y$-direction, viscous forces acting on the nozzle wall in $y$-direction, and from the natural reactive force of the transverse injection in 


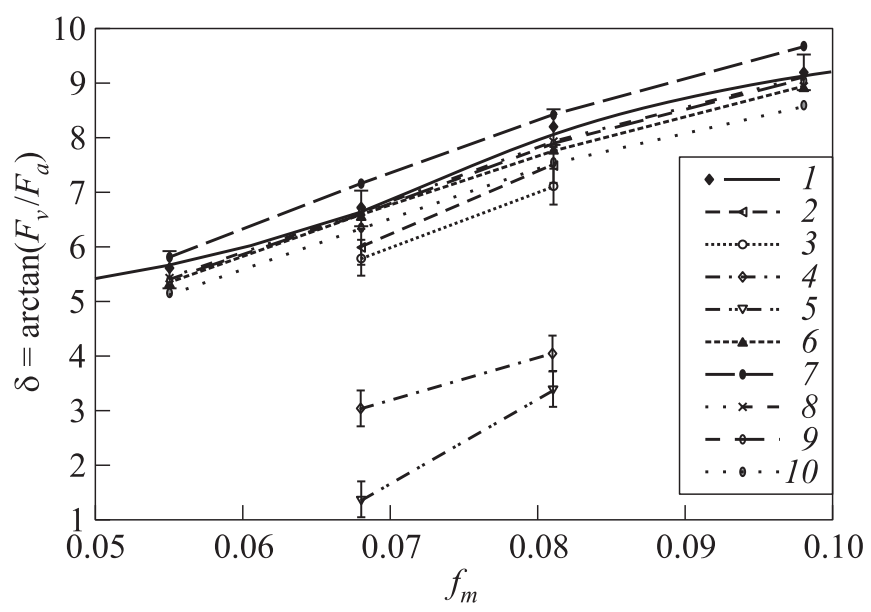

Figure 19 Thrust-vector pitch angle vs. mass-flow-rate ratio for different NPR ( $1-$ 37.5 (curve - numerical simulation, signs - experiments); $2-30 ; 3-15 ; 4-10$; and $5-7)$ and analytical models $(6-$ Schmucker (rocket nozzle, real gases); 7 Reshotko-Tucker (isentropique flow, $\mathrm{M}<3$ ); 8 - Green [17] (conical nozzle); 9 Schilling [16] (conical nozzle); and 10 - Kalt-Bendall (conical nozzle, cold air))

$y$-direction. Utilizing the constructed force-balance, the forces acting upon the nozzle are measured and data are coupled with the flow properties measurements to analyze the performances. The signal of the vertical transducers, where the vector side force is traced, is fluctuating between 0.61 and $1.01 \mathrm{~N}$ or $\sim 5 \%$. The range of standard deviation for axial thrust force was between 1.6 and $2.9 \mathrm{~N}$ or $\sim 2.3 \%$. The lateral transducer has high sensitivity and is used to inspect appearance of the asymmetric side loads. To examine performance of the shockvector system, the derived efficiency parameters are considered together with mass-flow-rate ratio and force vector angle (Table 2). The usual force coefficient:

$$
C_{F}=\frac{F_{R}}{F_{0}}=\frac{\sqrt{F_{x}^{2}+F_{y}^{2}}}{F_{0}}
$$

is substituted with the force efficiency parameter:

$$
\eta_{F}=\frac{I_{\mathrm{sp}}^{*}}{I_{\mathrm{sp}}}=\frac{\sqrt{F_{a}^{2}+F_{v}^{2}} /\left(\left(\dot{m}_{j}+\dot{m}\right) g_{0}\right)}{F /\left(\dot{m} g_{0}\right)}
$$

which is defined as the ratio between global specific impulses in vectoring and nonvectoring modes. 
Table 2 Experimental and numerical result-data of flow properties and force measurements

\begin{tabular}{|c|c|c|c|c|c|c|c|c|c|}
\hline \multirow{2}{*}{$\mathrm{SPR}=$} & \multirow{2}{*}{$\frac{p_{j}}{p_{c}} f_{m}=\frac{\dot{m}_{j}}{\dot{m}_{i}}$} & \multirow{2}{*}{$\begin{array}{c}F_{j y}, \\
\mathrm{~N}\end{array}$} & \multirow{2}{*}{$\begin{array}{l}F_{w y}, \\
\mathrm{~N}\end{array}$} & \multirow{2}{*}{$\frac{F_{v}}{\text { num. exp. }}$} & $\overline{F_{a}}$ & \multirow{2}{*}{$\begin{array}{c}\delta \\
\text { num. exp. }\end{array}$} & \multirow[t]{2}{*}{$\eta_{F}$} & \multirow[t]{2}{*}{$\overline{\eta_{T}}$} & \multirow[t]{2}{*}{$\overline{C_{V}}$} \\
\hline & & & & & exp. & & & & \\
\hline \multicolumn{10}{|c|}{ Case 1: $x_{j} / x_{t}=0.7$} \\
\hline$\overline{1}$ & 0.081 & 9.43 & -5.86 & 3.5724 .60130 .31 & 131.05 & $1.57^{\circ} 2.0^{\circ}$ & 0.954 & 0.94 & 0.197 \\
\hline \multicolumn{10}{|c|}{ Case 2: $x_{j} / x_{t}=0.9$} \\
\hline$\overline{0.667}$ & 0.055 & 6.63 & 6.04 & 112.6712 .4127 .76 & 126.8 & $5.67^{\circ} 5.6^{\circ}$ & 0.957 & 0.944 & 1.016 \\
\hline 0.833 & 0.068 & 8.02 & 6.92 & 14.9415 .0128 .39 & 128.3 & $6.64^{\circ} 6.7^{\circ}$ & 0.957 & 0.94 & 0.981 \\
\hline 1 & 0.081 & 9.88 & 8.30 & $18.18 \quad 18.4 \quad 128.5$ & 127.8 & $8.05^{\circ} 8.2^{\circ}$ & 0.949 & 0.921 & 0.947 \\
\hline 1.167 & 0.098 & 11.47 & 9.30 & 20.7720 .5128 .89 & 127.3 & $9.15^{\circ} 9.2^{\circ}$ & 0.937 & 0.91 & 0.93 \\
\hline
\end{tabular}

Remarks.

1. Basic nonvectoring case values: $\mathrm{NPR}=37.5, \dot{m}=223.2 \mathrm{~g} / \mathrm{s}, F=126.4 \mathrm{~N}$, and $I_{\mathrm{sp}}$ $=57.77 \mathrm{~s}$.

2. Num. - numerical, and exp. - experimental.

The axial thrust efficiency parameter represents the change in specific impulse in axial thrust direction which is related to the injectant mass-flow-rate part that augments the axial force:

$$
\eta_{T}=\frac{I_{\mathrm{sp} x}^{*}}{I_{\mathrm{sp} x}}=\frac{F_{a} /\left(\left(\dot{m}_{j}+\dot{m}\right) g_{0}\right)}{F /\left(\dot{m} g_{0}\right)} .
$$

Finally, the ratio of the vector-side force and axial force in term of vector pitch angle is compared with the mass-flow-rate ratio as the thrust vectoring parameter:

$$
C_{V}=\frac{\delta}{\left(\dot{m}_{j} / \dot{m}\right) \cdot 100} .
$$

These numerical and experimental results are presented in Table 2 with fixed adapted $\mathrm{NPR}=37.5$ and varied $\mathrm{SPR}$.

The numerical and experimental results are plotted together with approximated curves from analytical-empirical models in Fig. 19. Performance of the thrust vectoring is compared in terms of forces acting on the nozzle and derived efficiency parameters for varying NPR from adapted to high overexpanded with small $(0.068)$ to moderate $(0.098)$ mass-flow-rate ratios. The data are in very good agreement and as assumed vectoring force is quasi-linearly increasing with the mass-flow rate which is the dominant factor. The case with injection port placed deeper inside the nozzle proved as inefficient for thrust vectoring at adapted flow regimes but with satisfying value of global specific impulse which might be convenient for separation control of highly overexpanded nozzles. It is observable that SITVC demonstrates low detriment with increase of ambient 
pressure until separation inside the nozzle becomes dominant. After this value, the efficiency is rapidly decreasing.

\subsection{Numerical Results of the Truncated-Ideal-Contour Nozzle Test Model}

The TIC nozzle of the same NPR $=37.5$ and with exit angle of $5.3^{\circ}$ is numerically tested. The simulations are performed with normal and angular circular injection at $x_{j} / x_{t}=0.9$. The shockless flow from the TIC nozzle is imposing clearer profile in the separated region without weak compression shocks interfering with the shocks generated by the injection.

The circular injection with inclination from the nozzle axis has been investigated experimentally by Nielson et al. [19] and also by Masuya et al. [18]. Nielson et al. [19] performed the experiments on series of the circular sonic injections of

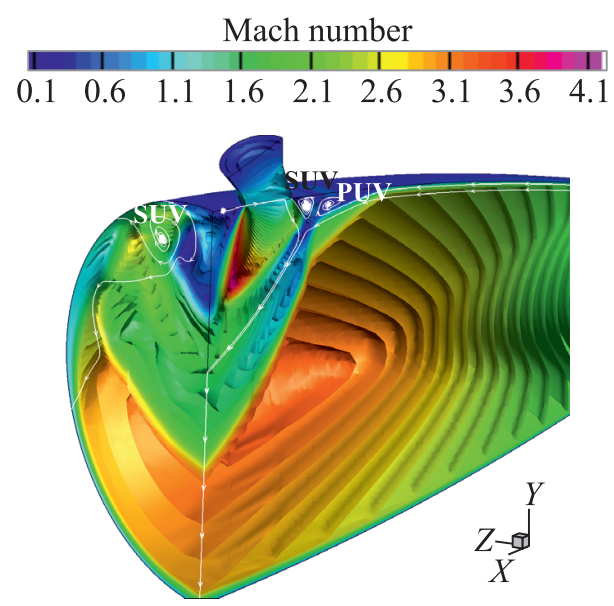

Figure 20 The 3D iso-Mach surfaces of circular injection with 20 degree upstream inclination from the normal position. $f_{m} \sim 0.06$ under different angles. It is found that downstream inclined injection has a negative effect on the side force while the upstream inclined angles positively affect the deflection until some limiting breakdown value. In Fig. 20 of the injection with an angle, it can be detected that leading edge of the barrel shock formed around the injectant plume is pushing shock-front head surface in the upstream direction and towards the nozzle axis. This extra push on the already separated flow is gaining steeper gradient to the deflection.

In order to response this extra blockage of the injectant, the main flow rapidly descends by the steep bow shock and circulate around the jet in spanwise direction. This action can be observed on the top plot of the wall pressure contours of normal and angular injection in Fig. 21. Wider separation zone with plateau region, PUV and SUV horseshoe vortex effectively turn the main jet while allowing still enough of unaffected flow to pass generating acceptable thrust force. To compare the data of the TIC and conical axisymmetric nozzle models, the force results together with derived performance parameters are given in Table 3 . The TIC nozzle has higher initial impulse but performance within vectoring mode is a bit higher with the conical nozzle. On other hand, 


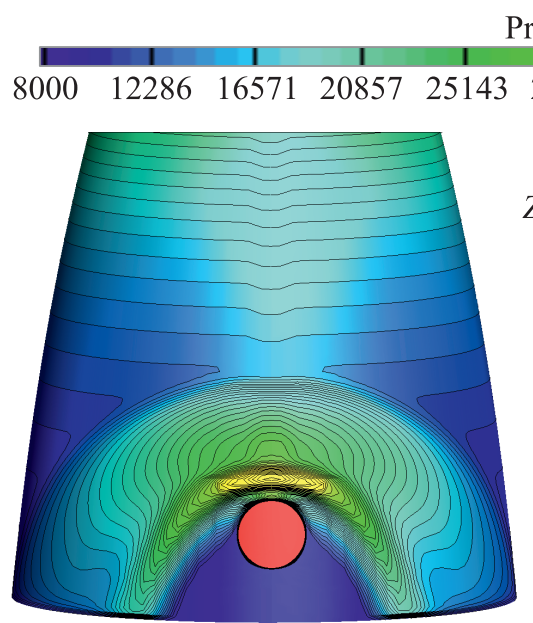

(a)

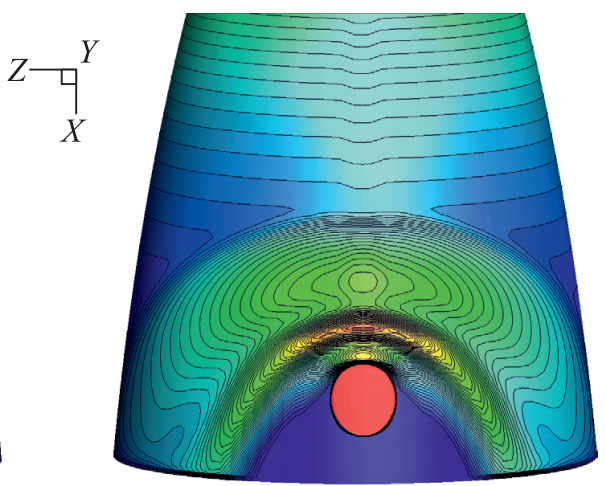

(b)

Figure 21 Normal $(a)$ and upstream inclined $(b)$ circular injections at $x_{j} / x_{t}=0.9$.

Table 3 Numerical result data of flow properties and force measurements of TIC and conical nozzle for $\mathrm{SPR}=1, \mathrm{NPR}=37.5$

\begin{tabular}{ccccccccc}
\hline Angle of secondary injection & Nozzle & $F_{i j}$ & $F_{w y}$ & $F_{v}$ & $F_{a}$ & $\delta$ & $\eta_{F}$ & $C_{V}$ \\
\hline \multirow{2}{*}{$0^{\circ}$} & TIC & 10.03 & 7.41 & 17.44 & 136.5 & $7.28^{\circ}$ & 1.026 & 0.983 \\
& Conical & 9.88 & 8.3 & 18.18 & 128.5 & $8.05^{\circ}$ & 0.949 & 1.012 \\
\hline \multirow{2}{*}{$20^{\circ}$} & TIC & 10.07 & 9.57 & 19.64 & 134.95 & $8.28^{\circ}$ & 0.944 & 1.035 \\
& Conical & 9.88 & 10.17 & 20.05 & 127.3 & $8.95^{\circ}$ & 0.943 & 1.1 \\
\hline \multirow{2}{*}{$30^{\circ}$} & TIC & 10 & 10.36 & 20.36 & 134.06 & $8.64^{\circ}$ & 0.938 & 1.08 \\
& Conical & 9.7 & 10.17 & 19.87 & 126.1 & $9^{\circ}$ & 0.934 & 1.111 \\
\hline \multirow{2}{*}{$40^{\circ}$} & TIC & 9.94 & 11.01 & 20.95 & 133.87 & $8.9^{\circ}$ & 0.936 & 1.113 \\
& Conical & 9.61 & 11.28 & 20.89 & 124.8 & $9.5^{\circ}$ & 0.926 & 1.172 \\
\hline
\end{tabular}

\section{Remarks.}

1. TIC basic nonvectoring values: $\dot{m}=235.9 \mathrm{~g} / \mathrm{s}, F=134.72 \mathrm{~N}$, and $I_{\mathrm{sp}}=57.9 \mathrm{~s}$.

2. Conical basic nonvectoring values: $\dot{m}=223 \mathrm{~g} / \mathrm{s}, F=126.4 \mathrm{~N}$, and $I_{\mathrm{sp}}=57.77 \mathrm{~s}$.

the part that augments the vectoring performance of conical nozzle degrades the thrust performance. The TIC and conical nozzles show very analogues performances with small difference of injection with $30^{\circ}$. This difference is due to the fact that weak compression wave of the conical nozzle ends just on the injection port and decreases performances of the SITVC.

Wall pressure profiles plot shown in Fig. 22 depicts the differences in the separation zones pressure distribution for the normal and upstream inclined injection. 


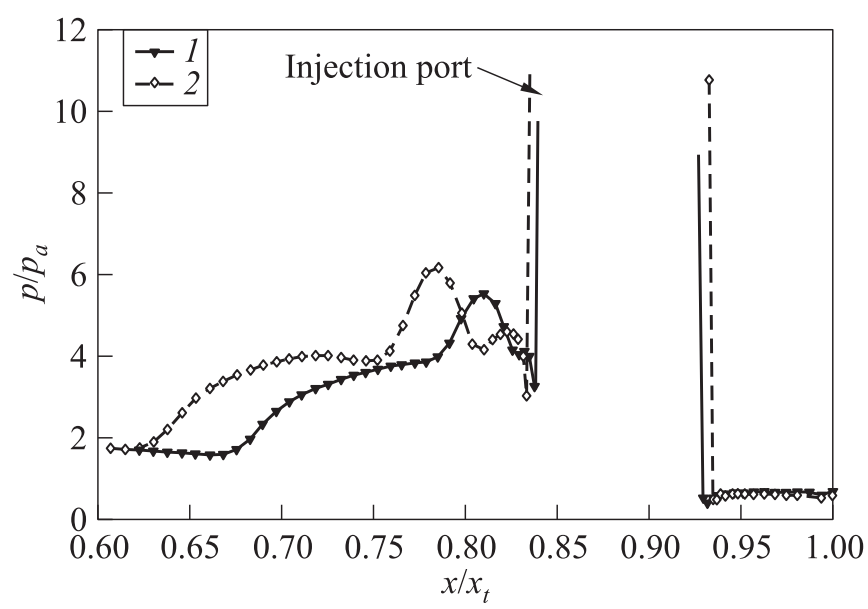

Figure 22 Wall-pressure profiles for normal (1) and 20 degree inclined injection (2) at $\mathrm{NPR}=37.5$ and $\mathrm{SPR}=1$

Action of the injectant plume on the separated flow displaces the separation point upstream and enlarges the separation region.

The zones of pressure rise in Fig. 22 are similar, but the zone dominated by the plateau pressure and PUV is considerably larger at inclined injection case. This is also followed by a quite larger zone of pressure peaks dominated by the SUV. As the secondary gas is injected under an angle, its overexpansion barrel shock leading edge acts as an aft and generates sudden pressure rise in the separated region larger traceable with the secondary pressure peak which occurs in this case.

To geometrically optimize the performances of SITVC, injection rate and performance of vectoring and thrust efficiency need to be balanced.

\section{CONCLUDING REMARKS}

Pertinent vectors side forces and pitch angles that are reached in the experimental and numerical tests of current investigation justified promising predictions for SITVC. The goal to examine general properties that influence shock vector control possibilities for C-D axisymmetric nozzles is accomplished with conical and TIC nozzles. A number of parameters such as the mass-flow rate, position and shape of the injector, angle of the secondary injection, and wide range of pressure ratios were investigated and their character and level of repercussion on the SITVC were determined. The used computational numerical method proved to be adequate to the supersonic cross-flow investigation. Big majority of the results was in excellent agreement with the experimental data and in 
correlation with analytically acquired profiles. The cases where multiple shocks and discontinuities are present at critical regions higher numerical resolution are needed. Numerically acquired results also complemented very well the valuable experimental result data obtained by the pressure transducers and force balance and together they demonstrated promising thrust vectoring possibilities on the supersonic conical nozzles test models.

The essential dissemblance with previously investigated 2D planar nozzle is depicted in terms of properties and physical nature of the flow in $3 \mathrm{D}$ nozzles. In $3 \mathrm{D}$ case, the strong bow shock propagates spanwise and in a flow direction. It is bounded and its spanwise propagation is restricted with the nozzle axisymmetric wall which further affects its shape. This physical process also lowers axial propagation comparing to the $2 \mathrm{D}$ case and allows more amount of unaffected main flow to be effectively exhausted maintaining the thrust specific impulse reduction at very satisfying range. With moderate mass-flow-rate ratio vector pitch angle ranging around $8.5^{\circ}$ is achieved enabling effective thrust vectoring. This can be further optimized and improved by altering the injection angle and injection port. The position of the injection port had decisive influence on the shock wave propagation and on the overall effectiveness of the vectoring system. Thus, the injection relatively closer to the nozzle exit proved to be more competent and effective. The other and conventionally used nozzle profiles such as truncated ideal contour are numerically tested and presented with their characteristics. The experimental test nozzles with this and other contours and injection geometries are in the process of investigation by the authors. More detailed wall pressure map will be obtained in experiments and complemented with a higher numerical resolution. This will allow further improvement in the study and analysis of the possible low-frequency instabilities and different separation modes.

\section{ACKNOWLEDGMENTS}

Special thanks to the French Space Agency (CNES) for collaboration and support and to Mr. Gouillon N. for technical assistance in this study.

\section{REFERENCES}

1. Neilson, J. H., A. Gilchrist, and C. K. Lee. 1968. Side thrust control by secondary gas injection into rocket nozzles. J. Mech. Engng. Sci. 10(3):239-51.

2. Mangin, B., A. Chpoun, and L. Jacquin. 2006. Experimental and numerical study of the fluidic thrust vectoring of a two-dimensional supersonic nozzle. AIAA Paper No. 2006-3666.

3. Wing, D. J., and V. J. Giuliano. 1997. Fluidic thrust vectoring of an axisymmetric exhaust nozzle at static conditions. ASME FEDSM97-3228. 
4. Deere, K. A. 2003. Summary of fluidic thrust vectoring research conducted at NASA Langley research center. AIAA Paper No. 2003-3803.

5. Waithe, K. A., and K. A. Deere. 2003. Experimental and computational investigation of multiple injection ports in a convergent-divergent nozzle for fluidic thrust vectoring. AIAA Paper No. 2003-3802.

6. Spaid, F. W., and E. E. Zukoski. 1968. Study of the interaction of gaseous jets from transverse slots with supersonic external flows. AIAA J. 6(2):205-12.

7. Zukoski, E. E. 1967. Turbulent boundary-layer separation in front of a forwardfacing step. AIAA J. 5(10):1746-53.

8. Schetz, F.W., and E. E. Billig. 1966. Penetration of gaseous jets injected into a supersonic stream. AIAA J. 3(11):1658-65.

9. Avduevskii, V.S., K. I. Medvedev, and M.N. Polyanskii. 1970. Interaction of a supersonic flow with a transverse jet injected through a circular aperture in a plate. Izvestiya Akademii Nauk SSSR, Mekhanika Zhidkosti Gaza 5:193-97. [In Russian.]

10. Santiago, J. G., and J. Dutton. 1997. Crossflow vortices of a jet injected into a supersonic crossflow. AIAA J. 35(5):915-17.

11. Chenault, C.F., and P.S. Beran. 1998. $k-\varepsilon$ and Reynolds stress turbulence model comparisons for two-dimensional flows. AIAA J. 36(8):1401-12.

12. Viti, V., R. Neel, and F. W. Schetz. 2009. Detailed flow physics of the supersonic jet interaction flow field. Phys. Fluids 21(4):046101-1-16.

13. Sellam, M., A. Chpoun, V. Zmijanovic, and V. Lago. 2012 (in press). Fluidic thrust vectoring of an axisymmetrical nozzle: An analytical model. Int. J. Aerodynamics.

14. Billig, E. E. 1967. Shock-wave shapes around spherical and cylindrical-nosed bodies. AIAA J. Spacecrafts 4(6):822-23.

15. Maarouf, N. 2008. Modelling study of dissymmetrical phenomena in the divergent of supersonic propulsion nozzles: Application to the thrust vectoring. Thesis dissertation. [In French.]

16. Schilling, T. W. 1962. Flow separation in rocket nozzles. M.S. Thesis. University of buffalo, New York.

17. Green, L. 1953. Flow separation in rocket nozzles. ARS J. 23(1).

18. Masuya, G., N. Chinzei, and S. Ishii. 1977. Secondary gas injection into a supersonic conical nozzle. AIAA J. 15(3):301-3.

19. Neilson, J.H., A. Gilchrist, and C.K. Lee. 1969. Control forces in rocket nozzles produced by a secondary gas stream inclined at various angles to the nozzle axis. J. Mech. Engng. Sci. 11(2):175-80.

20. Durand, P., B. Vieille, H. Lambare, P. Vuillermoz, G. Boure, P. Steinfeld, F. Godfroy, and J. F. Guery. 2000. CPS: A three-dimensional CFD numerical code dedicated to space propulsive flows. AIAA Paper No. A00-36976 3864.

21. Zucrow, M., and J. D. Hoffman. 1977. Gas dynamics. Willey and Sons.

22. Dussauge, J.-P. 2009. Compressible turbulence in interaction of supersonic flows. Springer Turbulence Interactions NNFM 105:35-54.

23. Guhse, R. D. 1966. On secondary gas injections into supersonic nozzles. AIAA J. $3(1): 147-49$. 